\title{
Le detto del Gatto lupesco : traduire l'intraduisible?
}

Il Detto del Gatto lupesco: tradurre l'intraducibile?

\section{Sylvain Trousselard}

\section{OpenEdition}

\section{Journals}

Édition électronique

URL : http://journals.openedition.org/cei/1332

DOI : $10.4000 /$ cei. 1332

ISSN : 2260-779X

\section{Éditeur}

UGA Éditions/Université Grenoble Alpes

\section{Édition imprimée}

Date de publication : 1 novembre 2013

Pagination : $37-55$

ISBN : 978-2-84310-234-9

ISSN : $1770-9571$

Référence électronique

Sylvain Trousselard, "Le detto del Gatto lupesco : traduire l'intraduisible? », Cahiers d'études italiennes [En ligne], 17 | 2013, mis en ligne le 01 mai 2015, consulté le 27 mars 2021. URL : http:// journals.openedition.org/cei/1332; DOI : https://doi.org/10.4000/cei.1332 


\title{
LE DETTO DEL GATTO LUPESCO : TRADUIRE L'INTRADUISIBLE?
}

\author{
Sylvain Trousselard \\ Université de Saint-Étienne
}

\section{Texte et traduction}

Si com' altr' uomini vanno, ki per prode e chi per danno, per lo mondo tuttavia, cosi m'andava l'altra dia per un cammino trastullando e d'un mio amor gia pensando e andava a capo chino. Allor uscio fuor del cammino ed intrai in uno sentieri ed incontrai duo cavalieri de la corte de lo re Artì, ke mi dissero: "Ki.sse' tu?" $E$ io rispuosi in salutare: "Quello k’io sono, ben mi si pare. Io sono uno gatto lupesco, ke a catuno vo dando un esco, ki non mi dice veritate. Però saper vogl[ $i]$ o ove andate, e voglio sapere onde sete e di qual parte venite." Quelli mi dissero: "Or intendete, e vi diremo ciò che volete, ove gimo e donde siamo; e vi diremo onde vegnamo. Cavalieri siamo di Bretagna, ke vegnamo de la montagna ke.ll'omo appella Mongibello. Assai vi semo stati ad ostello per apparare ed invenire
Comme les autres hommes vont, qui dans la joie, qui dans la peine, constamment, à travers le monde, ainsi allais-je l'autre jour par un chemin très plaisamment et pensant à un mien amour et je cheminais tête basse. Hors du chemin je sortis lors et pénétrai dans un sentier et rencontrai deux chevaliers de la cour du bon roi Arthur, qui me dirent : "Qui es-tu donc?" Je répondis en saluant : "Ce que je suis, se voit fort bien. Certes je suis un chat lupesque, qui à chacun donne une épreuve, si vérité il ne me dit.

Je veux savoir où vous allez, et veux savoir où vous naquîtes, de quelle contrée vous venez.» Ils répondirent : «Lors entendez, nous vous dirons à votre guise, où nous allons et d'où nous sommes; et vous dirons d'où nous venons. Nous sommes chevaliers Bretons, qui revenons de la montagne que l'on appelle Mont Gibel. Très longtemps y sommes restés pour découvrir et pour comprendre 
la veritade di nostro sire lo re Artù, k'avemo perduto e non sapemo ke.ssia venuto. Or ne torniamo in nostra terra, ne lo reame d'Inghilterra.

A Dio siate voi, ser gatto, voi con tutto "l vostro fatto." $E$ io risposi allora insuno: "A Dio vi comando ciascheduno." Cosi da me si dipartiro li cavalieri quando ne giro. $E$ io andai pure oltre addesso per lo sentiero ond'iera messo, e tutto 'l giorno non finai infin a sera, k'io albergai con un romito nel gran diserto, lungi ben trenta miglia certo; ed al mattino mi ne partio, si acomandai lo romito a Dio. Ed ançi k'io mi ne partisse, lo romito si mi disse verso qual parte io andasse: veritade non li celasse.

$E$ io li dissi: "Ben mi piace; non te ne serò fallace k'io non ti dica tutto 'l dritto. Io me ne vo in terre d'Egitto, e voi' cercare Saracinia e tutta terra pagania, e Arabici e 'Braici e Tedeschi [e..................... -eschi] e 'l soldano e 'I Saladino e 'l Veglio e tutto suo dimino e terra Vinençium e Belleem e Montuliveto e Gersalem e l'amiraglio e 'l Massamuto, e l'uomo per kui Cristo è atenduto dall'ora in qua ke fue pigliato e ne la croce inchiavellato da li Giudei ke 'l giano frustando, com' a ladrone battendo e dando. Allor quell' uomo li puose mente Esi li disse pietosamente:

"Va' tosto, ke non ti dean si spesso"; $e$ Cristo si rivolse adesso, sì li disse: "Io anderòe, e tu m'aspetta, k'io torneròe"; la vérité sur notre roi Arthur, que nous avons perdu et dont nous ignorons le sort. Or nous rentrons en notre terre, dans le royaume d'Angleterre. À Dieu, sieur chat, recommandons, toute votre bonne fortune. " Et je répondis aussitôt : "À Dieu vous recommande tous.» Ainsi s'éloignèrent de moi les chevaliers prenant la route. Et je partis bien au delà par le sentier où me trouvai, et tout le jour je ne cessai jusqu'au soir où je m'arrêtai chez un ermite, au grand désert, à bien trente milles de là ; et au matin je le quittai, recommandant l'ermite à Dieu. Et avant que je ne m'en aille, l'ermite, alors, me demanda vers quelle contrée m'en allais : que la vérité je ne cèle. Je répondis : "Bien volontiers; je ne serai pas fallacieux, je te dirai de tout le vrai. Je pars pour la terre d'Égypte, parcourir la Saracinie et toute la terre païenne, et arabe et juive et tudesque [et.........................-esque] et le sultan et Saladin et le Vieux et tout son domaine, terre des vivants et Bethléem Mont Oliviers, Jérusalem l'émirat, le Massamotin, et l'homme qui attend le Christ depuis qu'il fut emprisonné et qu'il fut cloué sur la croix par les Juifs qui l'ont fouetté, battu, rossé comme un voleur. Alors cet homme réfléchit et le pria très humblement : "Fuis à présent, échappe aux coups"; et le Christ alors lui parla, il dit ainsi : "Je partirai, et attends-moi, je reviendrai"; 
e poi fue messo in su la croce a grido di popolo ed a boce. Allora tremò tutta la terra: cosi.cci guardi Dio di guerra." A questa mi dipartio andando $e$ da lo romito acomiatando, a cui dicea lo mio vïag[g]io. Ed uscio fuor dello rumitag[g] io per un sportello k'avea la porta, pensando trovare la via scorta ond'io andasse sicuramente. Allor guardai e puosi mente e non vidi via neuna.

L'aria era molto scura, e 'l tempo nero e tenebroso; e io com' nomo päuroso ritornai ver' lo romito, da cui m'iera già partito, e d'una boce l'appellai, si li dissio: "Per Dio, se.ttu sai lo cammino, or lo minsegna, k'io non soe dond'io mi tegna." Quelli allora mi guardòe, co la mano mi mostròe una croce nel diserto, [lungi] ben diece miglia certo, e disse: "Colà è lo cammino onde va catuno pelegrino ke vada o vegna d'oltremare." A questa mi mossi ad andare verso la croce bellamente, e quasi non vedea neente per lo tempo ch'iera oscuro, $e$ 'l diserto aspro e duro. $E$ a l'andare k'io facea verso la croce tuttavia si vidi bestie ragunate, ke tutte stavaro aparechiate per pigliare ke divorassero, se alcuna pastura trovassero. Ed io ristetti per vedere, per conoscere e per sapere ke bestie fosser tutte queste ke mi pareano molte alpestre; si vidi un grande leofante ed un verre molto grande ed un orso molto superbio puis il fut placé sur la croix aux cris et la clameur du peuple. Alors trembla toute la terre : que Dieu nous garde ainsi du mal. » Lors je m'apprêtai à partir et de l'ermite prenant congé, à qui j'exposais mon voyage. Et je sortis de l'ermitage par un portillon de l'entrée, pensant trouver la droite voie où je puisse aller sûrement. Je regardai, je réfléchis et je ne vis aucune voie. L'air était devenu très sombre, et le ciel noir et ténébreux; et moi comme un homme peureux je m'en retournai vers l'ermite, dont je m'étais bien éloigné, et de ma voix je l'appelai, je lui dis : "Par Dieu, si tu sais le chemin, alors dis-le moi, car je ne sais par où aller.» L'ermite alors me regarda, Et de la main il me montra là une croix dans le désert, éloignée d'au moins dix bons milles, et dit : «Par là est le chemin où passent tous les pèlerins qui vont ou viennent d'Outre Mer.» Lors je m’apprêtai à partir vers cette croix, et de bon gré, et je ne voyais presque rien à cause du temps qui était sombre, et le désert âpre et dur. Sur le chemin que j'empruntais pour parvenir à cette croix je vis des bêtes réunies, toutes bien prêtes à l'affût pour attraper et dévorer, la moindre proie trouvée par là. Moi je restai pour observer, pour comprendre et pour découvrir quelles étaient toutes ces bêtes qui me semblaient vraiment sauvages; je vis ainsi un éléphant et un verrat vraiment très grand aussi un ours très orgueilleux 
ed un leone ed un gran cerbio; e vidivi quattro leopardi $e$ due dragoni cun rei sguardi; e si vidi lo tigro e 'l tasso e una lonça e un tinasso; e si vi vidi una bestia strana, ch'uomo appella baldivana; e si vi vidi la pantera e la giraffa e la paupera e l gatto padule e la lea e la gran bestia baradinera; ed altre bestie vi vidi assai, le quali ora non vi dirai, ké nonn-è tempo né stagione. Ma.ssi vi dico, per san Simone, ke mi partii per maestria da le bestie ed anda' via, e cercai tutti li paesi ke voi da me avete intesi, e tornai a lo mi' ostello. Però finisco ke.ffa bello. et un lion et un grand cerf; et j'y vis quatre léopards et deux dragons aux yeux cruels; j'y vis le tigre et le blaireau et une once et un tinasseau et j'y vis une étrange bête, que l'homme appelle baudouaine; et j'y vis aussi la panthère la girafe et la petite oie et le chapalu et la laie, la grande bête barathrum; et tant d'autres bêtes je vis, dont je ne vous parlerai là, car ici n'est point le moment. Mais je vous dis, par saint Simon, que je m'enfuis habilement et m'en allai d'entre ces bêtes, et parcourai tous les pays dont je vous ai tout raconté, et je rentrai en ma demeure. Je finis là : comme il convient.

\section{Présentation générale}

Le Detto del Gatto lupesco ${ }^{\mathrm{I}}$ reste, aujourd'hui encore, bien obscur pour la critique $^{2}$ en général. Il n'existe, en effet, aucune tradition manuscrite pour ce long poème anonyme de 144 vers qui ne figure que dans le manuscrit Magliabecchiano II.IV.III composé en I274 et renfermant divers traités moraux. Même pour les spécialistes du Moyen Âge certains textes posent problème, celui-ci en est la parfaite illustration. Le Detto del Gatto lupesco illustre cette constatation, car on ignore quand il a été rédigé précisément. La date de composition du manuscrit nous met sur la voie d'un texte du $\mathrm{XIII}^{\mathrm{e}}$ siècle, d'une part par sa forme qui rejoint celle d'autres poèmes et, de l'autre, par la cohérence qui caractérise la composition même du volume et le poème en question ${ }^{3}$.

I. L'édition de référence pour la présente étude est celle publiée par Annamaria Carrega, Il Gatto lupesco e il Mare amoroso, Alexandrie, Edizioni dell'Orso, 2000, 97 p.

2. L'édition d'Annamaria Carrega se fonde, pour l'essentiel, sur les travaux de Leo Spitzer, Luigi Sasso, Michelangelo Picone, Antonio Lanza et Guerrieri Crocetti.

3. Armando Petrucci, "Storia e geografia delle culture scritte (dal secolo XI al secolo XVIII)", dans Letteratura italiana: storia e geografia, éd. Alberto Asor Rosa, t. II, L'età moderna, Turin, Einaudi, 1988, vol. 2, p. II93-I292. 
Le poème présente le voyage, ou pseudo-voyage, d'un être se présentant sous les traits d'un animal indéfinissable, le «chat lupesque», et s'exprimant à la première personne. Loin d'incarner la voix collective à travers l'usage d'une première personne énonçante, il expose un parcours improbable, un voyage dont la logique et les contours nous échappent au fil de la narration. Face à ce texte pour lequel l'objectif initial a été la traduction, rien ne semble résolu et mes choix ont dû parfois se cristalliser dans des formes à minima pour respecter un choix métrique initial, celui de l'octosyllabe, et présenter un hapax dans la littérature italienne des origines et une dynamique nouvelle face à un texte qui restera problématique à bien des égards. Il s’agira donc de présenter brièvement des aspects de la diégèse afin de tenter une analyse des choix sémantiques, lexicaux et rythmiques de la traduction proposée. Tous ces éléments constituent le résultat d'une analyse du texte comme ouvre littéraire et se placent en amont et en aval de l'objectif initial qui est et reste la traduction dont la finalité, peut-être illusoire, est de lui donner un sens autonome, une existence indépendante, différente, dans un contexte lui-aussi autre.

La topique bretonne, tout d'abord, développée au début du récit, reste parfaitement obscure, car, dans l'économie générale du poème, elle n'occupe aucune place dans la diégèse et, d'une façon plus générale, la fonction référentielle reste, à mes yeux, inexistante. J'aurais tendance à prendre cette rencontre fortuite du locuteur déambulant et plongé dans des pensées amoureuses (dont on ignore d'ailleurs le contenu et la nature puisque l'auteur ne mentionne plus rien sur le sujet dans la suite du poème) comme l'occasion qu'il se donne pour se présenter en se nommant. Cet épisode breton serait davantage un leurre et une tentative vaine, mais certainement parodique, dont l'objectif serait d'insérer le poème qui débute dans une tradition pour mieux s'en éloigner si on souligne l'aspect fortuit de la rencontre et la disparition définitive, dans la suite du poème, de toute évocation de ces topoi.

L'épisode de l'énigme, quant à lui, que le Chat lupesque pose pour «laisser passer» les chevaliers est également surprenant. On aurait tendance à croire qu'il est en mesure de les bloquer, de les retenir s'ils n'obtempèrent pas, c'est-à-dire s'ils ne répondent pas à sa requête : c'est donc lui qui autorise le passage (cette caractéristique est d'ailleurs étonnante pour quelqu'un qui, ensuite, se perdra en quittant l'ermite chez qui il a

Pour Petrucci, le texte et le manuscrit révèlent «une exécution contemporaine presque absolue et une nette et très forte analogie textuelle et graphique» (p. I207). 
passé la nuit). Cette rencontre n'est donc que l'occasion d'une présentation double: le chat se nomme puis c'est au tour des chevaliers, justifiant au passage leur présence par leur quête. Il y a donc un clivage qui oppose le «chat» et les chevaliers. Le protagoniste se promène sans objectif précis en pensant à d'autres choses (v. 6-7: $e$ d'un mio amor già pensando / e andava a capo chino) et il suit un parcours non précisé (v. 5 : per un mio cammino trastullando) et sans but réel. C'est la rencontre qui fixe les deux groupes : ils se nomment successivement — comme nous l'avons souligné — et les chevaliers vont exposer les raisons de leur présence, leur provenance et leur destination. Ces deux éléments concernent des personnages qui disparaîtront du texte dès que le "chat lupesque» les aura quittés, mais ils sont les seuls à être véritablement cohérents dans le poème. Le protagoniste ne donnera, à aucun moment, la moindre justification de son parcours, de ce qui l'aura motivé et, enfin, de ce qui aura conditionné son retour. Tout oppose donc le «chat lupesque» aux chevaliers, à la fois dans le statut social et dans l'être même : ce sont des personnages que leur noblesse et leur courage ont placés au service d'un roi, Arthur en l'occurrence, dont les qualités ne sont pas à démontrer, car la simple évocation du nom de ce roi épargne au poète toute justification (la référence est commune à tous et suffisante). Face à eux se trouve un "chat lupesque» dont on ignore jusqu'à l'aspect physique, et prenant le dessus face à des personnages valeureux et nobles (si l'on en croit les qualificatifs qui les caractérisent et la tradition littéraire qui leur est consacrée).

À la différence des chevaliers dont le voyage a pour but la recherche du roi Arthur disparu depuis longtemps, le chat lupesque prend la route, seul, à la suite de cette rencontre fortuite. L'abandon des chevaliers précède un itinéraire dont on ignore les caractéristiques mais dont on apprend qu'il aboutit chez un ermite qui offrira le gîte au protagoniste. Sur ce court voyage d'une journée, n'est livrée aucune précision particulière. Aucun moyen de transport n'est mentionné, seul le point d'arrivée coöncide avec la fin de la journée donnant à la destination une dimension triple, temporelle et doublement topographique (v. 39-46) ${ }^{4}$. La distance de 30 milles, impossible à parcourir à pied en une journée, implique donc l'utilisation d'un moyen de transport mais qui n'est pas mentionné dans le texte. La seconde journée se présente comme un départ avorté provoquant le retour du protagoniste. L'ermite redevient l'hôte et se transforme en guide en indiquant le chemin à emprunter pour que le voyage puisse effectivement

4. v. 39-46: E io andai pur oltre addesso / per lo sentiero ond'iera messo, / e tutto 'I giorno non finai / infin a sera, $k$ 'io albergai / con un romito nel gran diserto, I lungi ben trenta miglia certo. 
débuter. À la suite de cet élément déclencheur, le chat lupesque entamera un voyage exagéré et improbable. Les moyens de transports, non signalés, deviennent implicites si on se réfère aux toponymes, aux distances parcourues, aux animaux rencontrés, aux personnages et aux contrées que le protagoniste évoque dans son récit. Le voyage ne sert pas un objectif spécifique puisqu'il ne s'inscrit pas dans une perspective d'évolution, de changement pour le protagoniste, seul le récit des péripéties occupe l'anecdote, comme un témoignage, un compte rendu objectivé dont la "vraisemblance», ou pseudo-vraisemblance, pourrait être rendue uniquement par un effet ininterrompu d'accumulations. C'est là une caractéristique majeure du poème, car seule la voix du protagoniste se fait entendre : elle constitue le seul canal par lequel transitent les informations. Le récit est ipso facto conditionné et l'objectivité du compte rendu se transforme en objectivation, si on juxtapose le déplacement géographique avec l'accumulation désordonnée présentée par le locuteur. Dans la diégèse, le récit du voyage futur ne laisse pas apparaitre une cohérence précise. Il se caractérise par un mélange confus entre toponymes et personnages rencontrés. Les premiers vers du poème sont même très éloignés d'un récit de voyage, puisque le protagoniste effectue une promenades. Il est plongé dans des pensées amoureuses et est étranger à toute préoccupation liée au périple qu'il entamera par la suite. À l'image de la déambulation initiale, aucun élément chronologique n'accompagne l'itinéraire qui se construit et le voyage ne tient pas compte de la moindre logique géographique. C'est alors la promenade qui prévaut, à l'image de la situation initiale, de la rencontre et de la station chez l'ermite. Cette pause, motivée par la fin de la journée, devient l'expression de l'égarement du personnage : elle se concrétise par la désorientation du locuteur. Les seuls développements susceptibles de constituer une démarche personnelle, un voyage raisonné, se transforment pour se réduire à un exposé prétendument taxologique où lieux et personnages se superposent et s'accumulent sans produire un sens précis et prédéfini. C'est la curiosité et, d'une certaine manière, la boulimie qui prennent forme et dominent un récit où le voyage disparaît au profit de l'énumération.

L'excipit ${ }^{6}$, enfin, apparaît comme une licence que s'octroie le poète. Le texte est une parodie de voyage initiatique, cela semble évident. L'auteur semble donc vouloir marquer, par ce point final, son choix de mettre un

5. v. 4-7 : cosi m'andava l'altra dia / per un cammino trastullando / e d'un mio amor già pensando / e andava a capo chino.

6. v. I44 : però finisco che fa bello («je finis là comme il convient»). 
terme à un récit où le voyage, comme nous l'avons dit, occupe une place pour le moins singulière dans l'économie générale du poème en se limitant à une énumération de toponymes, d'animaux et autres personnages. L'absence de construction cohérente produit de cette manière un effet d'accumulation dont on ignore à la fois le point de départ et le point d'arrivée. Dès lors, on est en droit de se demander quel est le réel objectif de ce pseudo-voyage sinon qu'il anticipe le "grand tour" tel que le pratiqueront les artistes et les jeunes aristocrates au XVIII ${ }^{\mathrm{e}}$ siècle pour se forger une culture. L'excipit est donc un ultime clin d'œil pour le lecteur afin qu'il puisse se rendre compte d'une forme de divertissement à la fois dans le contenu et dans la forme. Le Detto del Gatto lupesco ne peut entrer en aucune manière dans le cadre d'une proposition de vie ou même dans un système référentiel où l'expérience individuelle serait susceptible de renvoyer à un modèle ou encore de s'inscrire dans un cadre historique spécifique. Nous pouvons donc conclure qu'il s'agit d'un voyage isolé où est exclue toute visée d'exemplarité. C'est en cela que la critique a présenté le poème comme la parodie d'un voyage : elle relève notamment l'absence de situation initiale justifiant le déplacement (un quelconque élément déclencheur, une rupture de l'ordre) et exigeant un changement chez le protagoniste. Le récit se résume à une accumulation et l'excipit clôt un itinéraire inabouti tant dans sa forme que dans l'absence de toute finalité (un retour à l'ordre établi, une amélioration de l'état psychologique ou physique du protagoniste par exemple).

\section{Choix de traduction}

Le bestiaire, parallèlement au voyage effectué par le protagoniste, constitue une des caractéristiques majeures du poème, à commencer par le protagoniste lui-même qu'il est impossible de définir réellement.

L'épithète lupesco offre la possibilité d'une "non définition", car il se limite à désigner un "chat» avant tout, mais ayant certains traits propres au loup : lesquels cependant? Dans quelles proportions? Dans la traduction, tout comme en italien, le suffixe "-esque» indique une ressemblance, une manière d'être ou d'agir, une originalité dans un sens plus ou moins péjoratif ou laudatif. Cet espace "laissé libre», d'un point de vue sémantique, et lié à la définition du protagoniste, offre à l'auteur une liberté plus grande pour construire le récit qui débute. Dans le cas du choix de "chat lupesque», pour la traduction, qui reprend dans la forme le texte d'arrière-plan (ou texte source), le merveilleux s'inscrit de manière plus 
palpable et, surtout, plus explicite, pour le lecteur. Le protagoniste n'est pas un "chat-loup»: certes, il a des qualités permettant de l'assimiler au "loup», mais rien de plus. À cela s'ajoute le fait que rien dans le texte nous permet d'ajouter un sens, ou encore un effet de sens supplémentaire, à ce qualificatif. Dans la traduction, il y aura donc deux options : le choix d'un animal (le chat) ayant des attributs connus et repérables (ceux du loup) et un autre animal construit sur le modèle d'un mot-valise ou encore une création verbale annonçant la naissance d'un animal nouveau dont les attributs, inexistants ou encore "non définis" donneront une valeur supplémentaire, magique, merveilleuse ou alors effrayante. Il faut ajouter également que le personnage/ protagoniste se désigne comme "un gatto lupesco». Le choix de l'article indéfini tend à classer le personnage au sein d'une famille animale, un groupe défini et circonscrit ${ }^{7}$. Ce procédé indique alors une connivence (ou une pseudo-connivence comme on voudra) entre l'auteur et le lecteur, car il sous-entend que le destinataire du texte sera en mesure, immédiatement, d'entendre de quoi il s'agit alors qu'il n'en est rien, car le "Gatto lupesco» demeure avant tout un hapax dans la littérature italienne. Il le positionne néanmoins face au reste du monde mais dans un espace décalé, même si des formes similaires, ou plutôt assimilables, peuvent être repérées dans la tradition française, je pense notamment au "chat-loup» évoqué plus haut, mais aussi au «chapalou» par exemple.

La définition/ présentation est donc avortée dès l'évocation de l'être, puisque le personnage s'inscrit dans un imaginaire, dans un espace merveilleux qui ne fera que se confirmer au fil des vers et, particulièrement, à travers l'évocation d'autres animaux qui seront énumérés plus loin. Le qualificatif devient alors autre et place l'ensemble du récit dans une sphère où les repères tendent à disparaître. Même si le récit se fait à la première personne et s'apparente à celui d'un individu proprement humain qui pense et qui s'exprime, le clivage s'installe dès les premiers vers pour donner à l'ensemble du récit une coloration toute particulière : le voyage, les contrées et les personnages annoncés, s'éloignent de l'espace réel ou de la vraisemblance nécessaire pour que le récit puisse entrer dans un cadre didactique ou encore édifiant. C'est donc la présence du personnage qui, dès le début du poème, donne la tonalité spécifique au texte en le présentant comme la "promenade» d'un dilettantisme personnel que le protagoniste offre à son auditeur ou son lecteur. Pour ce qui est du "chat-loup",

7. C'est dans ce cas la fonction généralisante de l'article indéfini : il ne s'agit donc pas d'une forme de déictique comme pour l'article défini. 
il désigne le loup cervier (lequel désigne à son tour deux espèces de lynx: le lynx commun et le lynx $d u$ Canada). Il indique donc une espèce, un être spécifique dont la fonction dans le texte est renforcée d'ailleurs par le mot-valise qui le désigne. Mais, dans le texte, le substantif fonctionne également comme le déictique d'une personne, d'une réalité pragmatique. Il n'identifie pas un être mais le présente dans ce qui le caractérise : il est "chat» et il est "loup" à la fois. On peut cependant gloser sur la place du substantif "chat", intervenant en premier dans la désignation. Cela peut suggérer que l'être est d'abord "chat" puis «loup». Cela étant, la dimension merveilleuse n'est pas immédiatement évacuée, même si elle est fortement altérée. Aucun animal existant ne peut être qualifié de "chat-loup", la désignation inscrit donc le protagoniste dans un ensemble différent où les référents sont autres. Cela étant, on ignore lesquels. Cette solution était alors à exclure par la précision qu'elle conférait à la désignation. Le protagoniste n'est pas défini précisément, il est imposé par l'auteur, son existence devient donc indiscutable, seuls les référents sont écartés pour l'éloigner d'une réalité qui n'aura pas de place dans l'ensemble du récit sinon à travers les diverses évocations toponymiques et historiques nécessaires à un cadre désordonné, comme nous l'avons souligné plus haut.

Les autres évocations animales constituent des exemples encore plus éloquents car ce qui pourrait constituer des préfixes, suffixes (nous dirons affixes) ne nous renseigne en rien sur une éventuelle construction lexicale de la part de l'auteur. Le choix de traduction se révèle alors fort délicat : la recherche d'éventuels équivalents dans la tradition française s'est révélée infructueuse (mais je ne suis pas en mesure d'affirmer définitivement qu'il n'y en a pas). Par ailleurs, même si un équivalent avait existé, il fallait également qu'il puisse convenir au projet de traduction à la fois pour les sonorités et le rythme choisis. Nous étudierons quelques exemples extraits du texte afin d'illustrer cette spécificité.

il verre/ le verrat: la forme ver est attestée dès le début du XII ${ }^{\mathrm{e}}$ siècle en ancien français ${ }^{8}$. Soulignons également la discussion de plusieurs critiques autour de l'interprétation de uers en "ours» ou "verrat" dans le commentaire du Roland'. Le choix de verrat nous a semblé opportun par l'étymon commun.

la lonza l l'once (fém.) : désigne un grand félin d'Asie centrale, l'origine du mot est ancienne et peut correspondre aisément au choix lexical de

8. ver : verrat, sanglier. Voir Psautier d'Oxford, dans Libri Psalmorum versio antiqua Gallica, Oxford, éd. Fr. Michel, I860, LXXIX, I4 : li vers de la selve.

9. Il s'agit de la discussion autour du manuscrit d'Oxford, 727 et 732. 
l'auteur, notamment à travers la référence directe avec le nom de la première bête de l'Apocalypse selon saint Jean dont la première attestation se situe vers $1 \mathrm{II}^{\mathrm{IO}}{ }^{\mathrm{O}}$. Une traduction plus "courante» pourra être celle du lynx ou encore du léopard. La panthère est attestée dans le Trésor de Brunetto Latini ${ }^{\text {II }}$ La lonza fait référence à la luxure, la référence dantesque devient toute naturelle même si le texte est antérieur à la Comédie ${ }^{12}$.

il tinasso / le tinasseau : les origines proposées successivement par les critiques ne viennent pas régler une lecture difficile de ce mot dont on ignore finalement l'étymon exact, l'ambigüité n'est levée par aucune proposition. Pour Spitzer ${ }^{13}$, un lien pourrait être établi avec l'ancien français satinas (satanas également, mais d'autres variantes morphologiques coexistent) pour indiquer ici une bête féroce dont les traits ne sont pas définis, mais suffisamment suggestifs pour donner l'effet escompté. Picone ${ }^{\mathrm{I} 4}$, quant à lui, renvoie à la langue d'oïl et au tinas (botte) établissant ainsi un lien avec une ambiance de taverne propre à la production des jongleurs et autres chansonniers qu'il associe à notre dit. Cette dernière proposition ne peut en rien être satisfaisante, car son unique vertu est celle de rapprocher le texte d'une veine littéraire alors que, dans le cas présent, il s'agit de compléter un bestiaire que le locuteur vient de débuter. La question de la parodie du voyage se pose certes dès le début du poème, mais cette explication étymologique, peut-être pratique, ne peut convenir. Nous devons donc nous contenter de la proposition de Spitzer qui a le mérite de se conformer à la logique énumérative des vers pris en considération. La traduction en tinasseau m'a semblée naturelle en utilisant, pour faire écho au vers précédent, la même forme graphique et la finale en -eau (blaireau / tinasseau) pour donner un effet phonique clair et ne pas rompre avec le rythme de l'énumération en polysyndète de ce passage. Il s'agit donc d'une pure «fabrication», d'un néologisme de peu de valeur j'en conviens, car il ne renvoie à rien de connu ou d'envisageable, ni dans sa construction, ni dans sa suggestion phonique. La création devient donc gratuite et vient «combler» un manque qui finalement a fini par exister également en

IO. Bible de Jérusalem, Paris, Cerf, coll. "Pocket», I998, Apocalypse selon saint Jean, XIII, 2 : «La bête que je vis ressemblait à une panthère, avec les pattes comme celles d'un ours et la gueule comme une gueule de lion; et le dragon lui transmit sa puissance et son trône et un pouvoir immense.»

II. Brunetto Latini, Li livres dou Trésor, éd. critique par Francis J. Carmody, Genève, Slatkine Reprints, I998, Livre I, chap. CiciII, p. I68.

I2. Comédie, Enfer, I, 32.

13. Leo Spitzer, "Il Detto del Gatto lupesco», Giornale Storico della Letteratura Italiana, n' ${ }^{\text {I34, }}$ 1956, p. 7.

I4. Michelangelo Picone, "Giullari d'Italia. Una lettura del Detto del gatto lupesco", Versants, n 28, I995, p. 95 . 
italien. En effet, mon questionnement a été de savoir si un lecteur italien contemporain, même aguerri à la littérature des origines, serait prompt à livrer une définition ou une brève explication pour ce mot.

la baldivana / la baldivaine : la critique ${ }^{15}$ explique la forme de baldivana comme le travestissement du mot Baudouin présent dans le Roman de Renart et désignant l'âne. La fonction de ce mot serait alors celle de "rendre étrange» un animal existant. Ici encore, le choix de la création s'imposait, en suivant l'origine proposée par Spitzer et Picone. Baudouin, déictique de l'animal, devait ainsi se féminiser pour donner lieu à une transformation (on retrouve d'ailleurs la forme baldovino chez Cecco Angiolieri ${ }^{16}$, chez Onesto da Bologna ${ }^{17}$, dans un poème adressé à Cino da Pistoia, nous rencontrons la forme baldovin, et enfin chez Cino da Pistoia ${ }^{18}$, l'adjectif balduina qualifiant une personne de stupide). L'étrangeté évoquée par le locuteur au v. I29 (e si vidi una bestia strana) se cristalliserait de cette manière sur un animal assimilable à l'âne, sans doute aussi têtu, mais altéré dans son allure, subissant une déformation physique allant de pair avec son état psychologique unanimement reconnu comme idiot; l'opposition peut également s'établir en parallèle avec le cheval, son noble cousin, que la littérature médiévale a placé au niveau le plus haut. La variation morphologique peut ainsi s'expliquer par la position à la rime entre strana et baldivana, la création ferait alors écho à l'étrangeté annoncée se justifiant par le choix de la forme verbale. Mon choix s'est donc porté sur la création d'un mot à partir de la dénomination existante dans la tradition française, Baudouin pouvant se féminiser aisément pour surprendre et demeurer compréhensible. La graphie en baudonaine a, quant à elle, pour but de reprendre, de loin j'en conviens, celle du texte source.

la paupera l l'oie : la forme verbale est présentée comme la déformation burlesque de papera par la critique ${ }^{19}$. Là encore, la variation morphologique tend à donner une dimension étrange à l'animal par le verbe qui le désigne, comme s'il s'agissait d'activer une fonction nominaliste pour laquelle le nom tiendrait lieu de réel.

15. L. Spitzer, art. cité, p. 17 et M. Picone, art. cité, p. 96.

16. Cecco Angiolieri, Le Rime, a cura di Antonio Lanza, Rome, Archivio Guido Izzi, 1990, sonetto CVI, p. 210, v. I: Stando lo baldovino dentro un prato.

17. Onesto da Bologna, sonetto VIII a M. Cino da Pistoia, Sieste voi, messer Cin, se ben váadocchio (v. II : ch'a trarre un baldovin vuol lunga corda), dans Carlo Antonio Villarosa (éd.), Raccolta di rime antiche toscane, vol. II, Palerme, Giuseppe Assenzio, 1817, p. 370-37I.

18. Cino da Pistoia, Satira scritta a Dante, XXXVI (v. I2 : di cosi fatta gente baldüina), dans ibid., p. 306-307.

19. M. Picone, art. cité, p. 96. 
le gatto palude/le chapalu: diverses formes coexistent comme capalu ou capalus. Elles renvoient toutes à la tradition arthurienne ${ }^{20}$.

la lea l la laie: la traduction ne pose aucun problème en français contemporain puisque la forme laie existe et désigne depuis toujours la femelle du sanglier. Le mot leha, en ancien français, est déjà attesté en latin (lehas) en 800 dans le Capitulaire de Villis ${ }^{21}$.

la baradineral le barathrum: la critique ${ }^{22}$ présente la baradinera comme la déformation de baraterie en moyen français indiquant la tromperie (l'inganno). Cette proposition est justifiée par la proximité existante avec l'art $\mathrm{du}$ barattiere du jongleur, comme le rappelle Picone ${ }^{23}$. Cette proposition me semble toutefois fort insatisfaisante par le lien qui peut s'établir entre la «tromperie» et un quelconque animal, en l'occurrence un véritable hapax pour la littérature italienne. Mon choix s'est donc orienté vers les dictionnaires pour tenter de conserver une homophonie, même toute relative, entre le texte source et le texte cible. J'ai remplacé baradinera, dont la suggestion phonique est certes plus "douce» par les sonores et nasales composant le substantif, par Barathrum et donc conservé un même début du mot pour donner ensuite une connotation plus violente à la fois d'un point de vue phonique et d'un point de vue référentiel. Le Barahtrum désigne le gouffre, le précipice d'où l'on jetait les condamnés à Athènes, il désigne également les abysses marins et, par analogie, l'enfer et le monstre qui y séjourne. La violence sous-jacente ajoutée au texte d'arrière plan fait ainsi écho aux vers II3-II6 annonçant des bêtes sauvages ${ }^{24}$.

Les autres références utilisées par le poète renvoient à des toponymes ou des personnages fictifs ou non, mais l'enjeu n'est pas là. Il s'agit avant toute chose de positionner le texte dans un système référentiel large, très large même, pour donner au voyage une dimension surprenante et exceptionnelle pour la faire entrer dans la sphère parodique que les critiques ont exploitée pour cet improbable itinéraire du Gatto lupesco.

Mongibello (v. 27) / Mont Gibel: le toponyme est très diffus au Moyen Âge et se retrouve dans un très grand nombre de textes. Il désigne l'Etna

20. Voir Alfred Owen Hugues Jarman, Llyr du Caerfyrddin, Cardiff, I982, LXxxiı-I76 p. et Colette Dehalle, La bataille Loquifer, thèse de doctorat, Université d'Aix-Marseille I, 1979, 205 p.

2I. Capitulare de Villis vel curtis imperii, Göttingen, éd. R. Schneider, 1968, art. XL : «Ut unusquisque iudex per villas nostras singulares et lehas, pavones, fasianos, enecas, columbas, perdices, turtures, pro dignatis causa omnimodis semper habeant."

22. Luigi Sasso, "La questione del genere nel Detto del gatto lupesco", Reinardus, vol. II, I989, p. I5I.

23. M. Picone, "Baratteria e stile comico in Dante (Inferno, XXI-XXII)», dans Studi americani su Dante, a cura di G. C. Alessio e R. Hollander, Milan, Franco Angeli, 1989, p. 63-86.

24. V. II3-II6 : si vidi bestie ragunate, / ke tutte stavaro aparechiate / per pigliare ki divorassero, I se alcuna pastura trovassero. 
et le mot provient de l'arabe djebel signifiant «mont». On retrouve la référence chez Franco Sacchetti (Trecentonovelle, I64) par exemple, elle demeure très courante et perdurera jusqu'à une époque très récente.

Saladino (v. 6I) / Saladin: le personnage historique traverse tous les récit du Moyen Âge. On le retrouve dans le Novellino et le Décaméron par exemple en particulier dans la nouvelle dite "des trois anneaux".

Veglio (v. 62)/Vieux ou Vieux de la Montagne : là encore le Vieux de la Montagne (en arabe Cheikh el-Gebel) présenté comme le Chef de la secte des assassins ou fumeurs de Hachich a alimenté toute la production médiévale, qu'il s'agisse de la prose ou de la poésie. La référence renvoie à sa puissance, à la domination sans partage qu'il avait sur ses terres et à la violence avec laquelle il est représenté. L'évocation fonctionne donc de manière autonome pour un texte médiéval. Cela étant, elle peut devenir problématique pour un public contemporain.

Vinençium (v. 63) / Terre des vivants : est une déformation du latin de terra viventium et fait référence directement au psaume $27^{25}$.

Amiraglio (v. 65) / émir : le mot vient de l'arabe amür signifiant «commandant» et qui a donné directement émir. Le sens initial est celui de commandant de la flotte, notamment à l'époque normande en Sicile. Il n'y a donc aucun doute sur le sens à donner à ce terme, mais la signification pour un lecteur contemporain et la traduction émir en français provoquera sans doute une altération sémantique et ne renverra que vers un isotope lié à un dignitaire du monde arabo-musulman sans connotation navale spécifique et immédiate.

Massamuto (v. 65)/Massamotin: Massamuto désigne les Arabes présents en Andalousie au XII ${ }^{\mathrm{e}}$ siècle et par analogie l'Andalousie elle-même. Le terme massamutino (ou massamotino) est un substantif d'origine arabe, probablement dérivé de mașmùdï, la monnaie frappée en Espagne par les Almohadi, dynastie qui tire son origine par la tribu berbère des Mașmūda. Il s'agit du nom par lequel, dans des documents des XII et XIII ${ }^{\mathrm{e}}$ siècles, on désigne le bisant d'or: "Donna mi so' di pèrperi, d'auro massamotino" (Cielo d'Alcamo).

L'objectif de proposer la traduction d'un texte ancien dans un code linguistique différent pose problème à plus d'un titre : mon but reste celui de rester fidèle au texte le plus possible. Je ne suis pas auteur mais traducteur : le texte ne mappartient donc pas, il me faut le décoder, l'entendre, 
avant de l'encoder dans le code linguistique qui est le mien. La distance temporelle pourra heurter le lecteur : la traduction devra donc donner la possibilité d'accéder à des textes qui n'auraient pu faire l'objet d'une lecture faute d'édition disponible. La connaissance partagée, entre auteur et lecteur, qui se trouve biaisée par une différence de culture évidente, l'est à plus d'un titre : en tant qu'universitaires et chercheurs, nous sommes déjà restés perplexes, pour ne pas dire désarmés, face à des textes, des vers, des paragraphes et, plus généralement, des mots dont le sens échappe encore parfois à l'ensemble de la critique, passée et présente. Le clivage culturel sera l'écueil inévitable susceptible de provoquer des erreurs d'interprétation, car les référents ne sont plus les mêmes. D'où la nécessité de passer par un travail d'analyse, d'explication et de contextualisation du texte avant de procéder à sa traduction. Cette constatation ne s'applique pas exclusivement à un public de médiévistes avertis, mais à un public littéraire, lettré.

Quels choix s'offrent donc aux traducteurs sinon de tenter de soumettre une forme, inévitablement nouvelle, mais dont la teinte pourra rappeler l'origine du texte, à savoir un texte médiéval? Le choix d'archaïsmes, par exemple, dans le lexique et la syntaxe. Pour la syntaxe, certaines inversions peuvent donner au texte une teinte poétique (ou pseudo-poétique) comme c'est le cas au v. 6 (pensant à un mien amour) où l'emploi de l'adjectif possessif mien renvoie à une forme plurielle non évoquée du groupe nominal qui serait "une de mes amours». La forme si vérité il ne me dit (v. I7) est également intéressante, car l'inversion du complément d'objet autorise l'éviction du second membre de la négation et l'article défini du COD, permettant ainsi d'insister, dans la lecture, sur les deux axes essentiels du vers qui sont "vérité» et «dire». L'absence du pronom personnel sujet est quant à elle plus délicate. L'exemple très longtemps y sommes restés (v. 28) a été rendu possible par la présence immédiate de la personne énonçante et la répétition dont elle faisait l'objet dans le texte. Toute ambiguïté étant levée sur ce point, l'omission du pronom personnel sujet pouvait ainsi être envisagée, mais il faut souligner qu'elle reste problématique dans son utilisation en langue française car les formes verbales n'indiquent qu'assez rarement l'identité de la personne énonçante, d'où l'obligation de recourir à un pronom sujet tant à l'oral qu'à l'écrit. L'éloignement du complément d'objet direct de son verbe, je te dirai de tout le vrai (v. 55), permet aussi d'insister sur les deux éléments essentiels du vers : dire et vérité avec le choix lexical de "vrai» qui donne la dimension absolue telle qu'elle est établie en philosophie, et "vérité», concernant un énoncé, une réalité, donc une dimension plus réduite. Le choix lexical attribue une dimension 
plus assertorique à l'énoncé que l'inversion syntaxique rend poétique dans sa forme. Linversion sujet/verbe, alors trembla toute la terre (v. 79), très courante en poésie, permet de placer pareillement les éléments dans un ordre spécifique, privilégiant l'action sur l'objet de l'action et évitant une finale verbale abrupte propre au passé simple. On retrouve des inversions similaires avec les vers suivants et de l'ermite prenant congé (v. 82) et et il dit : "Par là est le chemin» (v. IO3). Parallèlement à ces quelques exemples, il est opportun de souligner certains usages qu'on rencontre fréquemment pour la traduction comme la transformation tantôt en asyndète, tantôt en polysyndète par exemple, que nous avons évoquée indirectement au gré de l'analyse des noms d'animaux traités plus haut. La présence de la conjonction de coordination $e$ débutant le vers a non seulement pour fonction d'associer les noms entre eux, mais de créer une surenchère dans l'accumulation, qui est de surcroît renforcée par l'effet anaphorique ainsi produit.

La construction syntaxique, même si elle subit une transformation significative, reprend une logique différente, l'asyndète pourra se substituer à la polysyndète ou les deux effets pourront alterner. Même si le traducteur s'éloigne du texte source par ces altérations, la recherche d'une cohérence dans le rythme pourra tenter de suppléer à ces légères divergences. L'objectif sera dès lors la cohérence au sein du texte cible. L'effet sera différent, certes, mais il répondra à la logique rythmique (dans le cas d'une traduction dont le choix d'un mètre sera une exigence de départ). Les inversions sujet/verbe deviennent, comme nous l'avons souligné plus haut, des choix intéressants pour la construction et l'élaboration des rythmes, des sonorités. Plus rarement, l'absence du pronom personnel sujet permet d'insérer une rupture syntaxique intéressante, non seulement pour le rythme, mais aussi pour l'effet qu'il provoque. Sa présence, en revanche, donne de cette manière l'effet d'une construction en zeugma, nominale ou pronominale cette fois, qui pourra mettre en relief l'élément essentiel du vers.

Parallèlement aux choix syntaxiques se mêlent également des choix lexicaux qui pourront donner au texte cible une teinte archaïque, ou archaïsante. L'exemple au v. 33 "Or ne torniamo in nostra terra" traduit en "Or nous rentrons en notre terre», ne pose pas de problème majeur pour la compréhension en dehors de la forme de ce Or qui pourrait surprendre et dont le sens de "à présent" pourrait se révéler altéré pour un public non averti ou aguerri face à une langue davantage classique. Ces choix se retrouvent in fine dans toutes les tentatives de traduction de textes anciens. Il semble difficile d'en faire l'économie. Même si cela relève du choix, la "médiété» sera de mise afin d'éviter tout excès dans un sens ou 
dans l'autre. Le travail de Jean-Charles Vegliante est éloquent sur ce point. Si nous prenons le premier tercet de la Divine Comédie, la choix lexical de sylve pour selva est attesté en français depuis le XII ${ }^{\mathrm{e}}$ siècle et se retrouve jusqu'au début du $\mathrm{Xx}^{\mathrm{e}}$ chez Valéry par exemple. Là encore le choix lexical ne surprendra pas le lettré, le spécialiste de littérature en langue romane, mais pourra heurter le lecteur moyen, mais cultivé, pour le choix de la forme adoptée. Ces choix pourront alors, peut-être, faire écho à certains néologismes pour lesquels il ne sera pas nécessaire de fournir d'explication car une traduction doit aussi tendre à un fonctionnement autonome (qui voudrait lire un poème pour lequel chaque vers, ou davantage, serait agrémenté d'une note explicative?) : nous connaissons l'exemple de la traduction de la Divine Comédie de Jean-Charles Vegliante qui fonctionne parfaitement et qui est dépourvue de la moindre note de bas de page. Sa formulation figurant dans la postface de La Comédie est éloquente sur le sujet : «je voudrais — plutôt qu'une postface, navrant pensum après un tel voyage! - suggérer quelques pistes de réflexion à partir du texte traduit (français), le mien, édité sans notes pour laisser la poésie souveraine de cette œuvre s'éployer aussi largement qu'il est possible sous le carcan d'un autre langage, et les limites du traducteur ${ }^{26}$.» Le point de vue du traducteur est ici clairement explicite et rend compte du statut qu'il confère à la traduction. Bien qu'elle soit étroitement liée au texte d'arrière-plan dont elle est la réécriture dans un code linguistique différent, il apparaît explicitement qu'elle se doit de fonctionner de manière autonome, comme l'expression, cette fois, du traducteur qui est le vecteur d'une expression autre, le passeur d'un «monde étranger» destiné à un public autre. Le texte «cible» devrait ainsi fonctionner de manière autonome, s'éloigner de sa source pour s'engager dans une existence personnelle en se substituant au texte qui aura provoqué sa gestation et provoqué sa naissance. Mais l'expérimentation de Jean-Charles Vegliante va plus loin, elle dépasse le simple texte à traduire pour donner vie à une forme nouvelle, peut-être renouvelée, du texte. Il est inutile de rappeler que la Divine Comédie a fait l'objet d'autres traductions, d'autres tentatives, voire d'expérimentations (le cas de la traduction proposée par André Pézard est emblématique sur ce point). Mais face aux créations verbales et aux rythmes imposés pour cette traduction, la traduction / création de Jean-Charles Vegliante n'aboutit pas, quant à elle, à un résultat obscur, le rythme y est clair et les choix plutôt transparents. La question qu'on serait en droit de se poser

26. Dante Alighieri, La Comédie, présentation et traduction de Jean-Charles Vegliante, Paris, Gallimard, 20I2, p. I2O7-I208. 
consisterait davantage à se demander quels sont les destinataires du texte ainsi renouvelé, mon travail de traduction du Dit du Chat Lupesque finit par se trouver dans une situation analogue.

Pour ma part, et pour conclure mon propos, le choix de l'octosyllabe s'est finalement imposé de manière assez naturelle car il correspond au mètre du récit, celui de l'épopée en l'occurrence. Le long poème rapportant les pérégrinations, ou pseudo-pérégrinations, du Chat lupesque, constitue un récit plus qu'un poème - je pense notamment au sonnet par exemple qui court vers sa fin et dont l'excipit constitue la pointe ou la chute. La régularité du mètre donne ainsi un rythme harmonieux facilement lisible pour une plus grande linéarité du texte. La rime cependant représentera la limite que je suis incapable de franchir. Le jeu des sonorités à l'intérieur des vers, quand elles apparaissent clairement, peut donner lieu à un certain nombre de tentatives (parfois heureuses mais, avouons-le, souvent infructueuses) destinées à donner une dimension supplémentaire au texte afin d'en livrer une saveur qui ajoutera un sens, une sensation dont l'objectif sera de s'inscrire au-delà du verbe, du message ou encore du référent. Une fois de plus, nous entrons dans le domaine de l'expérimentation, dans le cadre de la formule dite "heureuse», "ramassée», qui saura se conjuguer avec la syntaxe et le lexique. Les sonorités joueront un rôle suggestif qui pourra, s'il est possible de les mettre en place, renforcer le message du texte, lui donner une existence susceptible de donner à voir et à connaître une réalité disparue.

\section{Bibliographie}

Angiolieri Cecco, Le Rime, a cura di Antonio Lanza, Rome, Archivio Guido Izzi, I990.

Carrega Annamaria, Il Gatto lupesco e il Mare amoroso, Alexandrie, Edizioni dell'Orso, 2000.

Dante Alighieri, La Comédie, présentation et traduction de JeanCharles Vegliante, Paris, Gallimard, 2012.

Dehalle Colette, La bataille Loquifer, Thèse de doctorat, Université AixMarseille I, I979.

Jarman Alfred Owen Hughes, "Llyr du Caerfyrddin ", Proceedings of the British Academy, vol. 71, 1985, p. 333-356.

Latini Brunetto, Li livres dou Trésor, éd. critique par Francis J. Carmody, Genève, Slatkine Reprints, 1998. 
Petrucci Armando, «Storia e geografia delle culture scritte (dal secolo XI al secolo XVIII)", dans Alberto Asor Rosa (éd.), Letteratura italiana: storia e geografia, t. II, L'età moderna, Turin, Einaudi, I988, p. II93-I292. PICONe Michelangelo, "Baratteria e stile comico in Dante (Inferno, XXIXXII)», Studi americani su Dante, a cura di G. C. Alessio e R. Hollander, Milan, Franco Angeli, 1989, p. 63-86.

—, "Giullari d'Italia. Una lettura del Detto del gatto lupesco», Versants, $\mathrm{n}^{\circ} 28$, I995, p. 73-97.

SAsso Luigi, "La questione del genere nel Detto del gatto lupesco", Reinardus, vol. II, I989, p. I44-I53.

Spitzer Leo, "Il Detto del Gatto lupesco", Giornale Storico della Letteratura Italiana, $\mathrm{n}^{\circ} \mathrm{I} 34, \mathrm{I} 956, \mathrm{p} . \mathrm{I}-24$.

Villarosa Carlo Antonio (éd.), Raccolta di rime antiche toscane, vol. II, Palerme, Giuseppe Assenzio, I8I7. 